\title{
MOTIVACIÓN Y RENDIMIENTO ACADÉMICO EN FORMACIÓN PROFESIONAL. UN ESTUDIO PRELIMINAR EN EL CICLO FORMATIVO DE GRADO MEDIO AUXILIAR DE ENFERMERÍA
}

\author{
MOTIVATION AND ACADEMIC PERFORMANCE IN PROFESSIONAL \\ TRAINING. A PRELIMINARY STUDY IN THE AUXILIARY MIDDLE LEVEL \\ NURSING TRAINING CYCLE
}

\author{
JONATHAN PÉREZ DEL PINO* \& ANTONIO JOAQUÍN FRANCO-MARISCAL**1 \\ *IES José María Pérez Pulido. La Palma. Santa Cruz de Tenerife (España) \\ **UNIVERSIDAD DE MÁlaGA. DePARTAMENTO de DIDÁCTICA DE LAS CIENCIAS EXPERIMENTALES
}

(ESPAÑA)

\section{RESUMEN}

Distintas investigaciones sobre educación apuntan a la motivación como un elemento clave para una enseñanza de calidad. El objetivo de este trabajo es determinar la relación existente entre los factores asociados a la motivación que influyen en el aprendizaje (motivación extrínseca y motivación intrínseca) y el rendimiento académico de estudiantes del primer curso del Ciclo Formativo de Grado Medio de Auxiliar de Enfermería en función de su edad. La muestra estuvo formada por 48 estudiantes de edades comprendidas entre 16 y 46 años que cursaban dicho ciclo formativo en un centro público de Valencia. Dichos estudiantes respondieron la adaptación en castellano del cuestionario Échelle de Motivation en Éducation (EME-S) de Vallerand y colaboradores. La prueba U de Mann-Whitney mostró que la edad en este grupo no influye en el tipo de motivación que presenta y su relación con el éxito y el fracaso escolar. Además, se concluye que no existe una diferencia motivacional intrínseca estadísticamente significativa entre el alumnado de este ciclo formativo menor de 25 años y los que superan esta edad.

Palabras clave: motivación, rendimiento académico, Formación Profesional, Ciclo Formativo de Grado Medio de Auxiliar de Enfermería

\begin{abstract}
Different studies on education show that motivation is one of the keys to achieve an effective teachinglearning process. The objective of this paper is to determine the relationship between the factors associated with motivation that influence learning (extrinsic motivation, and intrinsic motivation) and the academic performance of students in Vocational Training according to their age. The sample consisted of 48 students of the first year of the Auxiliary Nurse Vocational Training from a public school in Valencia, aged between 16 and 46 years. These students answered the adaptation in Spanish of the Échelle de Motivation en Éducation (EME-S) questionnaire of Vallerand et al. The Mann-Whitney $U$ test showed that age is not an influencial factor in the type of motivation of the students and its relation to school success or failure in this group. In addition, there is no significant intrinsic motivational difference from the statistical point of view between the students of this training cycle under 25 years and those of older age.
\end{abstract}

Keywords: motivation, academic performance, Vocational Training, Auxiliary Nursing.

${ }_{1}^{1}$ Autor de correspondencia: Antonio Joaquín Franco-Mariscal. Email: anjoa@uma.es 


\section{Introducción}

Distintas investigaciones sobre educación apuntan a la motivación como uno de los aspectos clave para guiar a los estudiantes hacia un aprendizaje eficaz y de calidad y prevenir el abandono escolar (Lieury \& Fenouillet, 2016; Revelli et al., 2013; Tapia, 2005; Valenzuela et al., 2015). Sin embargo, esta motivación personal, que puede abarcar un amplio abanico de necesidades, se acentúa en las aulas de Formación Profesional (FP) donde el rango de edad de los estudiantes es tan amplio y las aspiraciones profesionales y ritmos de vida son tan distintos. Esta diferencia motivacional podría repercutir tanto en su rendimiento académico como en su interacción con los demás estudiantes (Tapia, 2003).

Entendiendo esta diversidad motivacional como necesidades especiales, la calidad de un proyecto curricular y de un centro se relacionará con su capacidad de atenderlas (Hernández, 2001). Así, un centro educativo de calidad será aquel que sea capaz de atender a la diversidad de individuos que aprenden (Garbanzo, 2007; Hernández \& Maquilón, 2010). En este marco, el profesorado juega un papel importante, ya que ante los cambios en la diversidad de edad que tiene hoy día el alumnado de FP, debe adaptarse en su metodología, pues los aspectos motivacionales varían mucho entre estudiantes menores de 20 años o mayores de 40.

Con estas ideas como punto de partida, el propósito de este estudio es determinar si la motivación que presenta el alumnado del primer curso del Ciclo Formativo de Grado Medio (CFGM) de Auxiliar de Enfermería de un instituto público de Valencia, varía en función de su edad, con una repercusión significativa en su rendimiento académico, para que sean tenidos en cuenta por los docentes en aras de conseguir el mejor rendimiento de los estudiantes. Esta investigación plantea un problema que llevaba observándose en las aulas los últimos años y que no había sido tratado en profundidad en la literatura en la etapa educativa de FP. A diferencia de otros estudios, el presente se centra en un ciclo formativo de la familia sanitaria en el que es clave despertar la vocación del alumnado y donde la diferencia de edad y por ende motivacional, suele ser muy acusada y determinante en el rendimiento académico.

\section{Fundamentación teórica}

\subsection{La motivación en el aprendizaje}

En el periodo de enseñanza secundaria obligatoria, la falta de motivación del alumnado es uno de los aspectos que puede condicionar el proceso de enseñanza-aprendizaje provocando bajos logros y abandono, evidencias de problemas que deberían dirigirse desde una perspectiva motivacional (Stover, De la Iglesia, Boubeta \& Fernández, 2012). Tapia (2003), sostiene que algunos estudiantes a pesar de contar con habilidades cognitivas que les permitan estudiar y aprender, se niegan a hacerlo. Esta creciente falta de interés provoca que manifiesten su aburrimiento por todo cuanto ocurre y se plantea en el ámbito escolar. Su preocupación pasa más por memorizar aquello que les permita aprobar que por aprender los conocimientos que les serán de utilidad en un futuro.

En esta línea, diversas investigaciones han mostrado que la motivación es un factor relevante en la predicción del rendimiento académico (Inglés, Martínez, Valle, García \& Ruiz, 2010), en la elección de futuros estudios (Shillingford \& Karlin, 2013) y en la prevención del fracaso escolar y el abandono prematuro de los estudios, siendo el docente capaz de potenciarla con su metodología y enfoque de las tareas (Gómez \& Toala, 2017).

El marco del Espacio Europeo de Educación Superior (EEES) se centra en el desarrollo de competencias y en la adopción por parte de los estudiantes de un rol más activo y autónomo, motivándoles a tomar sus propias decisiones, en el marco de una filosofía educativa constructivista, para aprender de manera autónoma competencias genéricas y específicas, para la profesión y para su propio proyecto vital (Martínez, 2011a,b). En este sentido, Anaya y Anaya (2010) afirman que el proceso de automotivación que lleve a cabo el propio estudiante en el 
contexto educativo del EEES es una genuina competencia y como tal, incluye determinadas formas de pensar, actuar, sentir y relacionarse.

La literatura recoge diferentes definiciones de motivación, coincidiendo diversos autores en que constituye el conjunto de procesos cognitivos, afectivos, conductuales y relacionales del estudiante implicados en la activación, dirección y persistencia de la conducta (Revelli et al., 2013), procesos en los que el contexto también juega un papel decisivo, además de la voluntad (Martínez, 2011a). Según Huertas (2006), para aprender es imprescindible "poder" hacerlo (componentes cognitivos), pero además es necesario "querer" hacerlo.

La teoría de la autodeterminación (TAD), que tiene su origen en los trabajos de Deci y Ryan $(1985,1991)$ y Ryan y Deci $(2000)$, autores que han contribuido notablemente al desarrollo de este campo de investigación, presenta una aproximación a la motivación humana que maximiza la importancia de los recursos internos para el desarrollo de la personalidad y las conductas autorreguladas y se apoya en aspectos sociales y ambientales que favorecen 0 disminuyen la motivación intrínseca (Núñez, Martín, Navarro \& Suárez, 2010; Vílchez, 2008). La TAD postula que la motivación puede ser expresada desde la falta de control hasta la autodeterminación (Núñez, Martín, Navarro \& Grijalvo, 2006; Núñez et al., 2010), con tres posiciones que reflejan la autonomía de los comportamientos: la amotivación, la motivación extrínseca (ME) y la motivación intrínseca (MI) (Stover et al., 2012). Cada una de estas posiciones presenta a su vez diferentes tipos (Tabla 1).

Tabla 1.

Tipos de motivación según TAD

\begin{tabular}{lll}
\hline TAD & & \\
\hline & (2) Motivación extrínseca (ME) & (3) Motivación Intrínseca (MI) \\
& (a) Regulación externa & a) Motivación al conocimiento \\
(1) Amotivación & (b) Introyección & b) Motivación al logro \\
& (c) Identificación & c) Motivación a las experiencias \\
\end{tabular}

La amotivación es un estado de falta de motivación que implica una percepción de incompetencia e incapacidad para actuar, ausencia de intención o de control para realizar una determinada conducta, poca o nula valoración de la tarea, sentimientos de indefensión y falta de expectativas para alcanzar el resultado deseado. En esta situación los sujetos no perciben que exista relación entre sus acciones y los resultados (Núñez et al., 2010).

En la ME la conducta tiene significado porque está dirigida a alcanzar una meta externa (Kuhl, 1992). Hace referencia a la participación en una actividad para conseguir recompensas y se define como un constructo multidimensional, donde se distinguen tres tipos que, ordenados de menor a mayor nivel de autodeterminación, son: regulación externa, introyección e identificación (Núñez et al., 2006). La regulación externa es el tipo más representativo de ME y se refiere a participar en una actividad para conseguir recompensas o evitar castigos; además, la conducta es el resultado de experimentar presiones externas o internas. En la introyección, aunque la conducta está regulada por exigencias o demandas, el individuo comienza a internalizar las razones de su acción, pero no está todavía autodeterminada; puede implicar coerción o presión, lo que impide al individuo tomar decisiones sobre su propia conducta. Por último, la identificación es el tipo de ME más autodeterminada, ya que el individuo valora su conducta y la cree importante; el compromiso es percibido como una elección propia, aunque se sigue considerando ME porque la conducta sirve para conseguir algo (Núñez et al., 2006).

Por su parte, la MI hace referencia al desarrollo de una actividad por la satisfacción inherente derivada de ella misma, es decir, que no precisa de reforzamientos externos y representa una tendencia innata de la naturaleza humana para buscar la novedad y el desafío, ampliar y ejercitar las capacidades propias y explorar y aprender (Wolters \& Pintrich 1998). Es el prototipo de 
conducta autodeterminada y se considera un constructo global en el que pueden ser diferenciados tres tipos: hacia el conocimiento, hacia el logro y hacia las experiencias estimulantes (Núñez et al., 2006). La MI hacia el conocimiento se ha relacionado con conceptos como la curiosidad o motivación para aprender y hace referencia a llevar a cabo una actividad por el placer que se experimenta mientras se aprende, o se intenta comprender algo nuevo. La MI hacia el logro puede ser definida como el compromiso en una actividad por el placer y satisfacción que se experimentan cuando se intenta superar un nuevo nivel. Finalmente, la MI hacia las experiencias estimulantes tiene lugar cuando alguien se involucra en una actividad para experimentar sensaciones positivas derivadas de ésta (Lamas, 2008).

Por otra parte, la literatura también recoge el término automotivación que se concibe como un proceso de autorregulación asociado al esfuerzo que dependerá del establecimiento de metas y de la percepción de autoeficacia por parte del alumnado, entre otras variables (Boekaerts, 1997; Marchago, 1992). Las metas elegidas actúan como estímulo del comportamiento, regulándolo y, se pueden situar entre dos polos que van desde una orientación extrínseca a otra intrínseca (García \& Doménech, 2000).

Como docente, debemos fomentar en el aula una MI de "dominio" aceptando retos y desafíos para incrementar los conocimientos y habilidades del alumnado, es decir, para afrontar una tarea por el hecho de aprender en sí y no por una 'recompensa', lo que libera al estudiante de las presiones de la competitividad (García \& Doménech, 2000) que solo busca obtener buenas calificaciones y no arriesgarse o ampliar e ir más allá de lo requerido. Trabajar intrínsecamente motivado posibilita incrementar capacidades, competencias y el disfrute de la tarea, por ende, el rendimiento académico del estudiante (Tapia, 2003), logrando superar aburrimiento y ansiedad y buscando soluciones por uno mismo. En resumen, debe trabajarse la confianza en sí mismos, fomentar la capacidad de logro, de aprender como fin último y poner unas metas concretas, realistas y alcanzables consensuadas con el alumno/a sobre las que poder trabajar con cierto margen y libertad para que la tarea que se va a llevar a cabo no aburra y produzca el cese de la actividad.

\subsection{Rendimiento académico}

Resulta oportuno relacionar los enfoques de aprendizaje con el rendimiento académico, al considerarse un indicador del nivel de aprendizaje que ha alcanzado el estudiante. Los profesionales de la educación conocen que la motivación es un elemento clave para el aprendizaje óptimo de un área curricular (Castelló \& Monereo, 2000). Por eso, hoy se entiende que los factores afectivos, dentro de los cuales se encuentra la motivación (García \& Musitu, 1993; Ruiz, Graupera, Gutiérrez \& Nishida, 2004), no pueden aparecer desvinculados de los factores cognitivos, tradicionalmente privilegiados en las aulas (Vivar, 2013).

De las investigaciones que relacionan enfoques de aprendizaje y rendimiento académico (Alonso \& Gallego, 1994) se desprende que la calidad del aprendizaje del alumnado depende de la calidad de enseñanza del profesorado. Cuando éstos son conscientes de sus enfoques de enseñanza (intenciones y estrategias) y tienen en cuenta los enfoques de aprendizaje de sus estudiantes se obtienen mejores resultados (Maquilón \& Hernández, 2011). Estudios recientes concluyen que existe una alta correlación entre las estrategias de apoyo que tienen como finalidad dar seguridad, incentivo y motivación al sujeto al afrontar las tareas de estudio, con el rendimiento académico del alumnado de secundaria. Además, éste será más bajo en la misma medida en que sea más alta la forma negativa de abordar las tareas (Barca, et al., 2013).

Ante el fracaso escolar, el papel del docente tiene un efecto positivo sobre el rendimiento del alumnado asociado a la Ml (Revelli et al., 2013). Será necesario un esfuerzo sistemático por estimular su interés presentando la materia en forma de mensajes significativos, relacionados con los intereses de los estudiantes y que les ayuden a desarrollar un pensamiento crítico (Alonso \& Gallego, 1994), ya que la finalidad de la educación es promover los procesos de 
crecimiento personal de cada individuo en el marco de la cultura del grupo al que pertenece (Castillo, Garabito \& Yahuita, 2006).

\section{Objetivos}

Esta investigación plantea dos objetivos: (1) Determinar la relación existente entre los factores asociados a la motivación que influyen en el aprendizaje (Ml y ME) y el rendimiento académico de estudiantes del primer curso del CFGM de Auxiliar de Enfermería en función de su edad, y (2) comprobar la existencia de diferencias estadísticamente significativas entre dichas relaciones.

Esta investigación pone su foco en una familia sanitaria de la FP por tratarse de una educación no obligatoria donde el alumnado acude por iniciativa propia, y donde debería reflejarse una mayor motivación en los estudiantes traducida en un buen rendimiento académico. Sin embargo, la realidad es muy diferente, encontrándose un alto absentismo, bajas de los ciclos y en muchos casos un deficiente rendimiento. En esta línea, la hipótesis que se pretende comprobar es que el alumnado de este estudio mayor de 25 años presenta una mayor MI por el aprendizaje, y esto repercute en un mayor rendimiento académico. Como segunda hipótesis se plantea que estas posibles diferencias debidas a la edad son estadísticamente significativas.

\section{Metodología}

Este estudio es de tipo descriptivo-correlacional no experimental (observacional), ya que se orienta a determinar el grado de relación existente entre las variables estudiadas (MI, ME, edad de los participantes y rendimiento académico) en una misma muestra de sujetos elegidos de forma no probabilística. La Figura 1 ilustra las distintas fases del estudio.

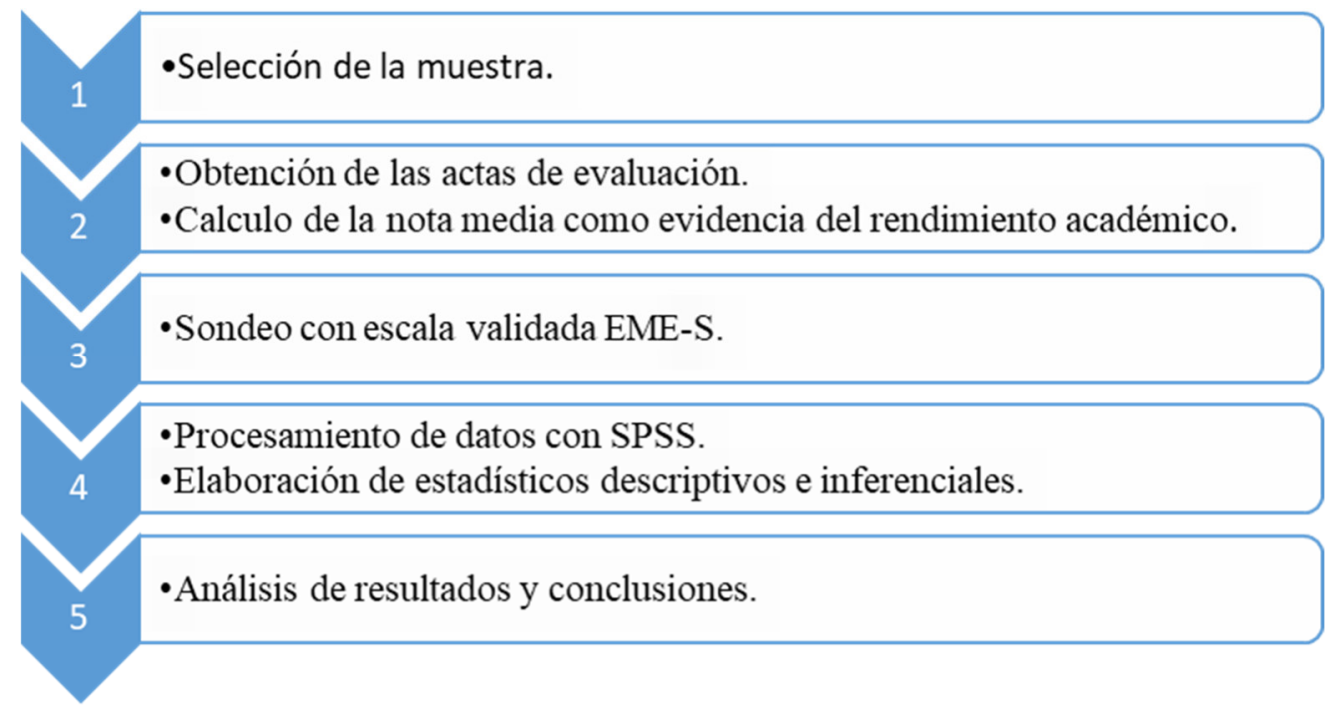

Figura 1. Fases del estudio

\subsection{Participantes}

La muestra de estudiantes fue de 48 sujetos, de los cuales el $73 \%$ eran mujeres $(\mathrm{N}=36)$ y el $27 \%$ hombres ( $N=13$ ), cuyas edades estaban comprendidas entre 16 y 46 años, siendo la edad media de 25.7 años y la desviación típica de 9.193. Todos ellos cursaban el primer curso del CFGM de Auxiliar de Enfermería en un centro público de Montcada (Valencia) en el curso 
escolar 2016-2017. Esta muestra no fue seleccionada al azar, sino por su accesibilidad al pertenecer todos al mismo ciclo formativo. Los participantes formaban parte de tres grupos (un grupo en horario de mañana y dos grupos de tarde). Su rendimiento académico medio fue de 6.26 puntos sobre 10 y se calculó como la nota media de los siete módulos de este ciclo correspondientes a la segunda evaluación en el citado curso. Las actas de las evaluaciones fueron cedidas por el profesorado tutor/a de cada grupo y se usaron para fines investigadores, manteniéndose en todos los casos la confidencialidad.

Para hacer los grupos de edad se partió del valor medio de 25 años de los participantes. La división de edades no fue homogénea, al encontrarse 28 estudiantes en el grupo de menores de 25 años y 20 sujetos en el grupo de $\geq 25$ años. Se observó que los estudiantes menores de 25 años tenían un rendimiento académico medio de 5.27 puntos, mientras que los $\geq 25$ años presentaban un rendimiento académico medio, recortado al $5 \%$, de 7.67 puntos, lo que originaba una diferencia de 2.8 puntos entre ambos grupos. Se decidió utilizar la media recortada al $5 \%$ porque dentro del grupo de $\geq 25$ años había un sujeto con valor atípico (nota media 3.85) ya que el resto de los sujetos de este grupo poseía medias superiores a 5.8.

\subsection{Instrumentos de recogida de datos}

Las características motivacionales iniciales de cada estudiante se valoraron a través de una adaptación del cuestionario Échelle de Motivation en Éducation (EME) propuesto por Vallerand et al. (1989), que se apoya en la TAD, y validado en español por Núñez, Martín-Albo y Navarro (2005). Dicha adaptación en castellano está enfocada al contexto de la educación secundaria postobligatoria (EME-S) (Núñez et al., 2010) y se compone de 28 ítems (ver Anexo I), distribuidos en siete subescalas que corresponden a los tres grados de autonomía en los que se basan los comportamientos según la TAD (Tabla 1).

El cuestionario refleja qué causas motivan más al alumnado y cuáles menos respondiendo a la pregunta ¿por qué vas al instituto? Las respuestas de cada subescala se puntuaron de acuerdo con una escala tipo Likert de cuatro puntos: 1 (muy en desacuerdo), 2 (poco de acuerdo), 3 (muy de acuerdo) y 4 (totalmente de acuerdo). Una valoración de 4 puntos corresponde con la puntuación más alta en dicho ítem, mientras que 1 implica la puntuación baja. Las puntuaciones mínima y máxima que se pueden obtener en el apartado de amotivación son 4 y 16 puntos, respectivamente, mientras que para Ml y ME, al tener tres tipos cada una, las puntuaciones mínima y máxima son de 12 y 48 puntos, respectivamente. Para la fiabilidad del cuestionario se calculó el estadístico alfa de Cronbach, obteniéndose un valor de .903, lo que otorga un alto grado de fiabilidad al instrumento.

Los datos obtenidos se analizaron con el programa SPSS 17.0. Para los dos primeros análisis se empleó la prueba $U$ de Mann-Whitney para muestras independientes no paramétricas, al tratarse de relaciones entre variables cuantitativas ( $\mathrm{MI}, \mathrm{ME}$ y rendimiento académico) y variables cualitativas (edad). Se aplicó también la prueba estadística inferencial de Levene para evaluar la igualdad de varianzas. Para el análisis confirmatorio de correlación lineal entre el rendimiento académico y la variable Ml (ambas variables cuantitativas) se utilizó el coeficiente de correlación de Spearman. En este estudio se consideran diferencias estadísticamente significativas si $p<.05$.

\subsection{Procedimiento y análisis de datos}

Se solicitó la colaboración del equipo educativo para aplicar el cuestionario EME-S al alumnado en una sesión de clase. Los estudiantes fueron informados del objetivo de la investigación y se les comunicó que la participación era voluntaria y confidencial con el fin de maximizar la validez de la información obtenida. Para asegurar la confidencialidad de sus datos personales y confirmar su voluntariedad en el estudio, se les entregó un consentimiento informado. No recibieron remuneración económica por su participación en el estudio. 
El análisis estadístico se centró en estudiar los tipos de motivación que influyen en el aprendizaje. Concretamente, se han realizado tres análisis: (a) la relación que existe entre los tipos de motivación ME y Ml y los grupos de edad, (b) entre el rendimiento académico de los estudiantes y los grupos de edad, y (c) entre la Ml y el rendimiento académico.

Los datos obtenidos se analizaron con el programa SPSS 17.0. Para los dos primeros análisis se empleó la prueba $U$ de Mann-Whitney para muestras independientes no paramétricas, al tratarse de relaciones entre variables cuantitativas (MI, ME y rendimiento académico) y variables cualitativas (edad). Se aplicó también la prueba estadística inferencial de Levene para evaluar la igualdad de varianzas. Para el análisis confirmatorio de correlación lineal entre el rendimiento académico y la variable Ml (ambas variables cuantitativas) se utilizó el coeficiente de correlación de Spearman. En este estudio se consideran diferencias estadísticamente significativas si $p<.05$.

\section{Resultados}

\subsection{Diferencias entre tipos de motivación (ME y MI) y edad}

La Tabla 2 recoge los resultados del cuestionario EME-S referidos a la ME y MI en función del grupo de edad.

Tabla 2.

Media y desviación típica de ME y Ml en la escala EME-S para los grupos de edad

\begin{tabular}{ccccc}
\hline & \multicolumn{2}{c}{ ME } & MI \\
\hline Grupo de edad (años) & Media & Desviación típica & Media & Desviación típica \\
\hline$<25(\mathrm{~N}=28)$ & 35.68 & 6.183 & 33.18 & 6.722 \\
$\geq 25(\mathrm{~N}=20)$ & 37.85 & 6.964 & 40.40 & 7.266 \\
\hline
\end{tabular}

Al comparar los resultados de cada grupo de edad (Tabla 2 y Gráfico 1), ambas en una escala de 12 a 48 puntos, se observa que no existen diferencias apreciables entre ambos grupos en cuanto a ME (diferencia de 2.17 puntos entre las medias y .781 para desviación típica). En cuanto a Ml, se observa una diferencia de 7.22 puntos entre las medias y de .54 puntos para la desviación típica.
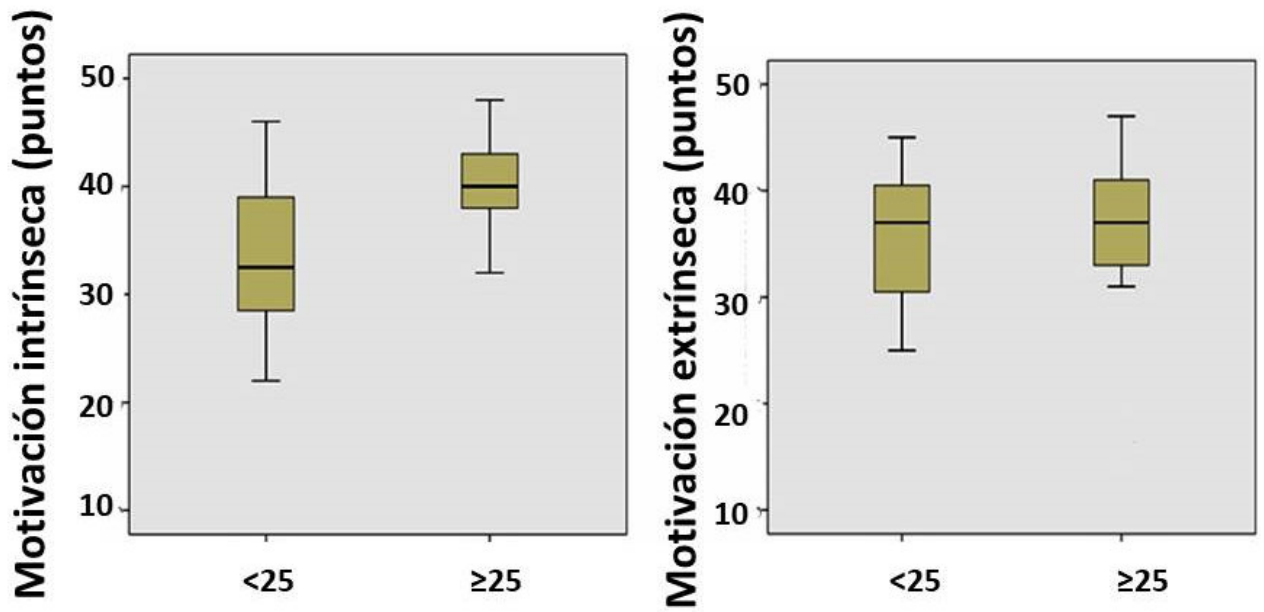

Grupo de edad (años)

Gráfico 1. Grupos de edad y MI (izqda.) y grupos de edad y ME (drcha.)

Estos estadísticos descriptivos podrían indicar que existe una relación entre la edad, la Ml y el rendimiento académico. Para comprobarlo se realizó la prueba $U$ de Mann-Whitney y de Levene 
para muestras independientes entre tipo de motivación y grupos de edad. La prueba $(\mathrm{U}=$ 232.500; $p>.05$, para Ml; $U=273.000 ; p>.05$, para ME) mostró que se debía conservar la hipótesis nula tanto para MI como ME de que la distribución de estos tipos de motivación es la misma entre las categorías de edad. En otras palabras, no existen diferencias estadísticamente significativas entre los valores medios de $\mathrm{Ml}$ y pertenecer a un grupo u otro de edad, ni tampoco entre los valores medios de ME y los grupos de edades.

\subsection{Diferencias entre rendimiento académico y edad}

Los resultados de la prueba $U$ de Mann-Whitney entre los grupos de edad y el rendimiento académico medio $(\mathrm{U}=338.500 ; \mathrm{p}>.05)$ confirmaron que tampoco existían diferencias estadísticamente significativas entre el hecho de pertenecer a un grupo u otro de edad y los valores medios del rendimiento académico.

\subsection{Relación entre Ml y rendimiento académico}

Previo al análisis confirmatorio de relación lineal se realizó una representación gráfica de dispersión del rendimiento académico de cada estudiante frente a su edad. Dicha representación (Gráfico 2) mostró una clara relación lineal ascendente entre ambas variables, lo que significa que existía una relación y que es positiva, es decir, a mayor edad, mayor nota media obtiene el alumnado en la evaluación considerada para el estudio.

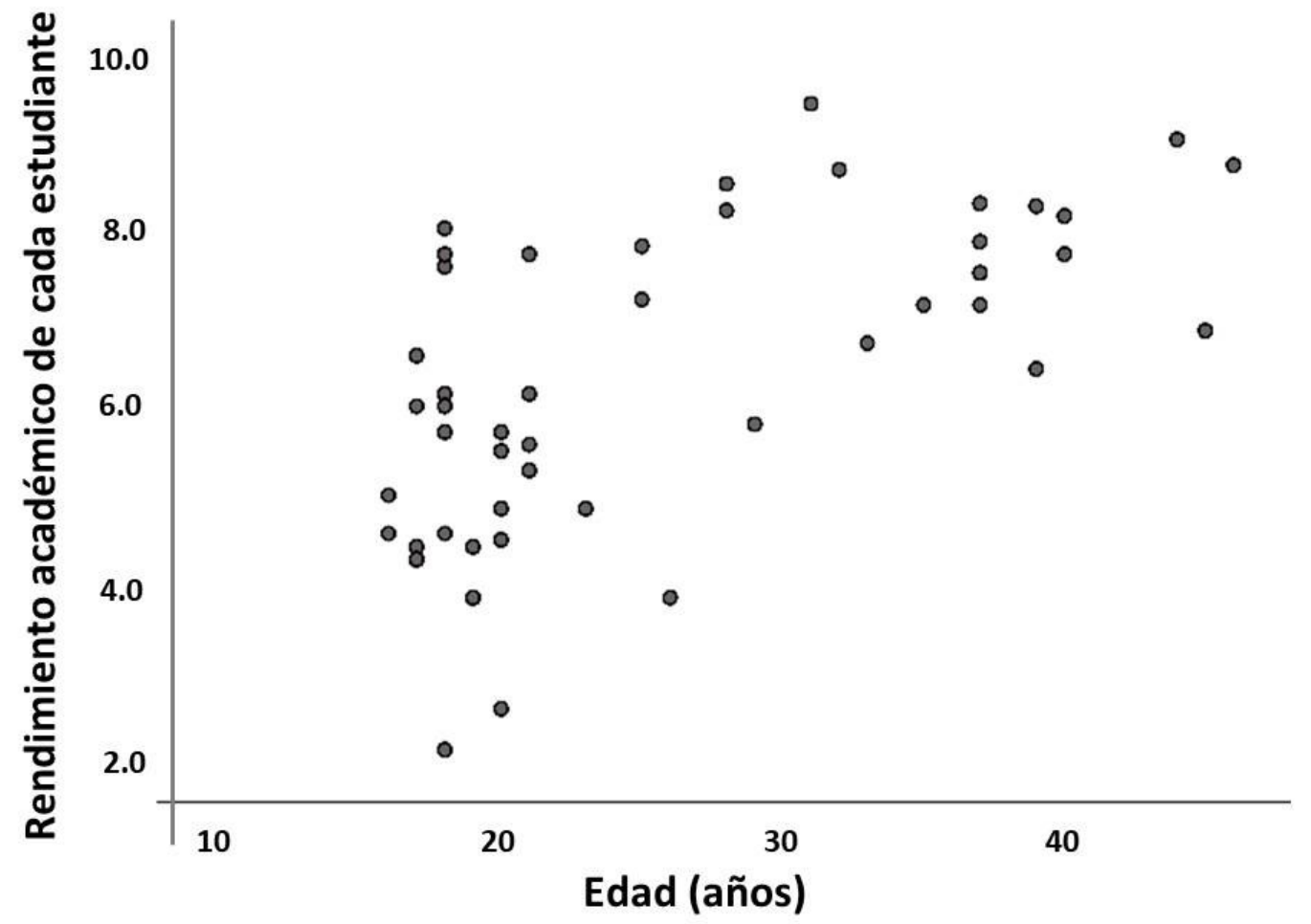

Gráfico 2. Gráfica de dispersión del rendimiento académico frente a la edad

La correlación de Spearman entre MI y la nota media mostró que existía una relación entre ambas variables (Rho de Spearman $=.230$ ), no siendo esta correlación estadísticamente significativa $(p>.05)$. Esto indica que en este grupo de estudiantes no existía una relación entre la edad, la Ml y las calificaciones obtenidas. 


\section{Discusión y conclusiones}

De la investigación desarrollada empleando el cuestionario EME-S se concluye que no existe una relación entre los diferentes tipos de motivación para el aprendizaje (MI y ME) y su rendimiento académico en función de la edad en los estudiantes del primer curso del CFGM de Auxiliar de Enfermería del centro educativo estudiado.

De forma más específica, la prueba $U$ de Mann-Whitney no encontró diferencias estadísticamente significativas entre estudiantes menores de 25 años y los que superan esta edad y su motivación hacia el aprendizaje (ya sea MI o ME), ni tampoco entre el rendimiento académico y la edad de los sujetos. No obstante, la prueba de correlación de Spearman comprobó la existencia de una relación lineal positiva entre Ml y rendimiento académico, aunque dicha relación no fue estadísticamente significativa.

Estos resultados rechazan la hipótesis de partida de que estudiantes de mayor edad en este ciclo presentan mayor $\mathrm{Ml}$ por el aprendizaje que se pone de manifiesto con mejores calificaciones escolares, al menos, en la muestra de estudiantes de la familia sanitaria con los que se realizó esta investigación. Asimismo, no se confirman los resultados de otros estudios sobre motivación en el contexto académico (Martínez, 2011b), en los que obtenían mejor rendimiento académico aquellos estudiantes con estructuras de motivación enfocadas al logro (MI). Esto plantea la necesidad de realizar un estudio similar a mayor escala y con mayor número de sujetos en la familia profesional sanitaria ya que, al tener una muestra pequeña, los datos no terminan de ser relevantes.

La principal implicación educativa de este estudio recae en conseguir motivar a los estudiantes con el objetivo de mejorar su aprendizaje y, por tanto, su rendimiento académico, atendiendo tanto a la MI como a la ME. Para este fin, es preciso que el profesorado utilice estrategias que aprovechen las propias motivaciones de los estudiantes (Clavel \& Balibrea, 2010; Díaz \& Hernández, 2002). La concepción constructivista de la educación asume que el aprendizaje significativo es en sí mismo motivador porque el alumnado disfruta realizando la tarea o trabajando esos nuevos contenidos, pues entiende lo que se le enseña y le encuentra sentido (Beltrán, 1993; Mc Robbie \& Tobin 1997). Cuando el estudiante disfruta de la tarea se genera una MI donde pueden aflorar una variedad de emociones positivas placenteras (Ruiz et al., 2004), que en última instancia repercutirán sobre el rendimiento académico del alumnado.

En ese sentido, para que el alumno/a se sienta motivado para aprender unos contenidos de forma significativa es necesario que los docentes sean capaces de atribuirle sentido (utilidad) a aquello que se enseña, activamente a los estudiantes en un proceso de construcción de significados (García \& Musitu, 1993), donde la meta se centre en el progreso y la mejora individual, donde los errores formen parte del proceso de enseñanza y aprendizaje (Hernández \& Maquilón, 2010).

Teniendo en cuenta esto, debe revalorizarse el papel del docente como mediador del aprendizaje (Gómez \& Toala, 2017), que debe utilizar estrategias constructivistas que aprovechen las propias motivaciones del alumnado (Clavel \& Balibrea, 2010; Ruiz et al., 2004) y fomenten la interacción mediante un aprendizaje cooperativo (Díaz \& Hernández, 2002).

Este trabajo más profundo de las estrategias de enseñanza y aprendizaje, focalizado en la motivación del alumnado, conllevará no solo a una mejora del rendimiento académico, sino también de la calidad educativa con la correspondiente reducción del fracaso escolar. Es por ello que los docentes deben dedicar tiempo y esfuerzo para que sus estudiantes adquieran las estrategias necesarias de estudio que contribuyan a un adecuado desarrollo de la motivación que conduzca a unos mejores resultados académicos. 


\section{Limitaciones del estudio y líneas futuras}

Debido a la falta de medios materiales y humanos, este estudio sobre la motivación en la familia profesional sanitaria presenta ciertas limitaciones que no garantizan que sus resultados sean extrapolables a toda la población. En primer lugar, hay un sesgo muestral de omisión, ya que el estudio se hizo solamente con estudiantes de un CFGM de la familia en particular.

Somos conscientes de que la muestra no es plenamente representativa debido al sesgo de inclusión que presenta, porque los participantes fueron seleccionados por conveniencia, dependiendo de su asistencia el día de la encuesta. Además, esta investigación posee un posible sesgo de medición debido a que los sujetos son muy reacios a dar respuestas socialmente inaceptables, por temor a ser juzgados y tienden a la deseabilidad social. Del mismo modo, los participantes pueden, consciente o inconscientemente, dar una respuesta que piensa que el entrevistador quiere oír. En futuros estudios se pretende aumentar el número de participantes para obtener datos más significativos extrapolables a toda la población. Para ello, se ampliará el estudio a todas las ramas de la FP y se estudiará qué ciclos son más sensibles a los problemas de motivación.

Otra posible línea de trabajo es el estudio del efecto de la metodología sobre la motivación empleada en el aula. En este sentido, teniendo en cuenta las necesidades motivacionales, sería interesante desarrollar una metodología de enseñanza basada en la puesta en práctica de los conocimientos teóricos, de forma que todo lo aprendido sea contextualizado por el estudiante. En este marco, la evaluación debe centrarse en los propios logros del estudiante y dejando un margen de autoevaluación, donde el alumno/a aprenda a ser crítico consigo mismo.

Sin olvidar que la motivación educativa no es una técnica o método de enseñanza particular, sino un factor cognitivo presente en todo acto de aprendizaje (Castillo et al., 2006) y que además depende en gran medida de factores como la edad, el sexo, la inteligencia, la situación social y los rasgos de la personalidad de cada estudiante (competitividad, curiosidad) así como sus aspiraciones, para motivar, se deberían tener en cuenta una serie de acciones por parte del docente, como promover los procesos de crecimiento personal del alumnado en el marco de la cultura del grupo al que pertenece (Mc Robbie \& Tobin, 1997), donde lo más importante no es la enseñanza sino el aprendizaje (Beltrán, 1993).

\section{Referencias}

Alonso, C., \& Gallego, D. (1994). Estilos de aprendizaje. En F. Rivas (Ed.), Manual de asesoramiento y orientación vocacional (pp. 56-89). Madrid: Síntesis.

Anaya, A., \& Anaya, C. (2010). ¿Motivar para aprobar o para aprender? estrategias de motivación del aprendizaje para los estudiantes. Tecnología, Ciencia, Educación, 25(1), 514.

Barca, A., Barca, E., Peralbo, M., Porto, A. M., \& Santorum, R., \& Vicente, F. (2013). Estrategias de aprendizaje, autoconcepto y rendimiento académico en la adolescencia. Revista Galego Portuguesa de Psicoloxía e Educación, 21(1), 195-212.

Beltrán, J. (1993). Procesos, estrategias y técnicas de aprendizaje. Madrid: Síntesis.

Boekaerts, M. (1997). Self-regulated learning: A new concept embraced by researchers, policy makers, educators, teachers, and students. Learning and Instruction, 7(2), 161-186.

Castelló, M., \& Monereo, C. (2000). Las concepciones de los profesores sobre la enseñanza de las estrategias de aprendizaje. Ensayos y Experiencias, 6(3), 78-92.

Castillo, V., \& Garabito, R., \& Yahuita, J. (2006). Estrategias docentes para un aprendizaje significativo. Cuadernos Hospital de Clínicas, 51(1), 96-101. 
Clavel, J. G., \& Balibrea, J. (2010). Motivación y rendimiento académico: Los intangibles de la educación. Investigaciones de Economía de la Educación, 5, 139-154.

Díaz, F. B. A., \& Hernández, G. R., (2002). Constructivismo y aprendizaje significativo. Estrategias docentes para un aprendizaje significativo, una interpretación constructivista ( $2^{\mathrm{a}}$ ed.) México: Mc Graw Hill.

Deci, E. L. \& Ryan, R. M. (1985). Intrinsic motivation and self-determination in human behavior. New York: Plenum Press.

Deci, E. L., Vallerand, R. J., Pelletier, L. G. \& Ryan, R. M. (1991). Motivation in education: the self-determination perspective. The Educational Psychologist, 26, 325-346.

Garbanzo, G. M. (2007). Factores asociados al rendimiento académico en estudiantes universitarios, una reflexión desde la calidad de la educación superior pública. Revista de Educación, 31(1), 46-63.

García, F. J., \& Doménech, D. (2000). Motivación, aprendizaje y rendimiento académico. REME, Revista Electrónica de Motivación y Emoción, 1, 55-65.

García, F. J., \& Musitu, G. (1993). Rendimiento académico y autoestima en el ciclo superior de EGB. Revista de Psicología de la Educación, 4(11), 73-87.

Gómez, M. E., \& Toala, C. L. (2017). La motivación del docente y su aporte en el rendimiento académico de los y las estudiantes de la escuela de educación general básica Justino Cornejo, del recinto el pueblito del Cantón 24 de mayo. Unpublished manuscript.

Hernández, F. (2001). La calidad de la enseñanza y el aprendizaje en educación superior. Revista de Investigación Educativa, 19(2), 465-489.

Hernández, F., \& Maquilón, J. J. (2010). Las concepciones de la enseñanza. Aportaciones desde la investigación. Revista Electrónica Interuniversitaria de Formación del Profesorado, 13(3), 17-25.

Huertas, J. A. (2006). Motivación: Querer aprender. (2ª ed.). España: Aique.

Inglés, C. J., Martínez, A. E., Valle, A., García, J. M., \& Ruiz, C. (2010). Conducta prosocial y motivación académica en estudiantes españoles de educación secundaria Obligatoria. Universitas Psychologica, 10(2), 451-465.

Kuhl, J. (1992). A theory of self-regulation: Action versus state orientation, self discrimination and some applications. Applied Psychology: An International Review, 41(2), 97-129.

Lamas, H. (2008). Aprendizaje autorregulado, motivación y rendimiento académico. Liberabit, 14(14), 15-20.

Lieury, A. \& Fenouillet, F. (2016). Motivación y éxito escolar. Argentina: Dunod.

Maquilón, J., \& Hernández, F. (2011). Influencia de la motivación en el rendimiento académico de los estudiantes de formación profesional. Revista Electrónica Interuniversitaria de Formación del Profesorado, 14(1), 81-100.

Marchago, J. (1992). Eficacia del feedback en la modificación del autoconcepto académico. Revista de Psicología General y Aplicada, 45, 63-72.

Martínez, J. A. (2011a). Diseño y validación del cuestionario de automotivación AM-24 en el marco de espacio europeo de educación superior. Cuadernos de Educación y Desarrollo, 3(27), 1-15.

Martínez, J. A. (2011b). Automotivación y rendimiento académico en el espacio europeo de educación Superior. Cuadernos de Educación y Desarrollo, 3(28), 1-13.

Mc Robbie, C., \& Tobin, K. (1997). A social constructivist perspective on learning environments. Internacional Journal of Science Education, 19(2), 193-208.

Núñez, J. L., Martín-Albo, J., Navarro, J. G., \& Grijalvo, F. (2006).Validación de la escala de motivación educativa (EME) en Paraguay. Interamican Journal of Psychology, 40(3), 391398.

Núñez, J. L., Martín-Albo, J., \& Navarro, J. G. (2005). Validación de la versión española de la échelle de motivation en éducation. Psicothema, 17(2), 344-349. 
Núñez, J. L., Martín-Albo, J., Navarro, J. G., \& Suárez, Z. (2010). Adaptación y validación de la versión española de la escala de motivación educativa en estudiantes de educación secundaria postobligatoria. Estudios de Psicología, 31(1), 80-100.

Revelli, J.A., Gutiérrez, P., Del Castillo, F., Centeno, M., Vinuesa, A., Belcaid, B., \& Andrade, M. T. (2013). Autoeficacia docente, motivación intrínseca y expectativa de resultado del alumnado. Reidocrea, 2, 54-62.

Ruiz, L. M., Graupera, J. L., Gutiérrez, M., \& Nishida, T. (2004). El test AMPET de motivación de logro para el aprendizaje en educación física: Desarrollo y análisis factorial de la versión española. Revista de Educación, 335, 195-211.

Ryan, R.M., \& Deci, E. L. (2000). La teoría de la autodeterminación y la facilitación de la motivación intrínseca, el desarrollo social, y el bienestar. American Psychologist, 55(1), 6878.

Shillingford, S., \& Karlin, N. J. (2013). The role of intrinsic motivation in the academic pursuits of nontraditional students. New Horizons in Adult Education and Human Resource Development, 25(3), 91-102.

Stover, J. B., De la Iglesia, G., Boubeta, A.R., \& Fernández, M. (2012). Academic motivation scale: Adaptation and psychometric analyses for high school and college students. Psychology Research and Behaviour Management, 5, 71-83.

Tapia, J. A. (2003). Motivaciones, expectativas y valores relacionados con el aprendizaje. Análisis empírico e implicaciones para la mejora de la actuación docente en la enseñanza secundaria y bachillerato (Tesis Doctoral). Madrid: Servicio de Publicaciones de la Universidad Autónoma.

Tapia, J. A. (2005). Motivaciones, expectativas y valores-intereses relacionados con el aprendizaje: El cuestionario MEVA. Psicothema, 17(3), 404-441.

Valenzuela, J., Muñoz, C., Silva, I., Gómez, V., \& Precht, A. (2015). Motivación escolar: Claves para la formación motivacional de futuros docentes. Estudios Pedagógicos (Valdivia), 41(1). http://dx.doi.org/10.4067/S0718-07052015000100021.

Vallerand, R.J., Blais, M.R., Brière, N.M., \& Pelletier, L.G. (1989). Construction et validation de l'Échelle de Motivation en Éducation (EME). Canadian Journal of Behavioral Sciences, 21, 323-349.

Vílchez, A. (2008). Test psicométrico para medir el grado de motivación intrínseca. Escuela de Post Grado de la Universidad Nacional "Pedro Ruiz Gallo" Lambayeque. Perú.

Vivar, M. (2013). La motivación para el aprendizaje y su relación con el rendimiento académico en el área de inglés de los estudiantes del primer grado de educación secundaria (Tesis Doctoral). Perú: Universidad de Piura.

Wolters, Ch. \& Pintrich, P. (1998). Contextual differences in student motivation and self-regulated learning in mathematics, english and social studies classrooms. Instructional Science, 26, $27-47$. 


\section{Anexo I: Cuestionario Escala de motivación en secundaria EME-S}

\section{Encuesta sobre motivación educativa}

La presente encuesta busca conocer las razones que te motivan a ir al instituto. Se te presentan 28 frases para que las leas atentamente y las respondas con sinceridad según sea tu grado de acuerdo o desacuerdo con ellas.

Marca con un aspa $(X)$ la alternativa que creas que más se acerque a tu opinión. No hay respuesta correcta ni incorrecta, no se trata de un examen. Recuerda que todo lo que expreses será tratado de forma confidencial.

\begin{tabular}{|c|c|c|c|c|c|}
\hline \multicolumn{6}{|l|}{ Nombre y apellidos } \\
\hline Edad & & & Sexo & & \\
\hline \multicolumn{4}{|c|}{ ¿Tienes asignaturas pendientes? } & Sí & No \\
\hline ¿Estudios previos? & Sí & No & ¿Cuáles? & & \\
\hline
\end{tabular}

\begin{tabular}{|c|c|c|c|c|c|}
\hline $\begin{array}{l}\text { Tipo de } \\
\text { motivación }\end{array}$ & ¿Por qué vas al instituto? & MD & PA & MA & TA \\
\hline $2 a$ & $\begin{array}{l}\text { 1. Porque necesito, al menos, el título del Ciclo para encontrar un trabajo } \\
\text { bien pagado }\end{array}$ & & & & \\
\hline $3 a$ & 2. Porque siento placer y satisfacción cuando aprendo nuevas cosas & & & & \\
\hline $2 c$ & $\begin{array}{l}\text { 3. Porque creo que haber cursado el Ciclo me ayudará a prepararme mejor } \\
\text { para la profesión que he elegido }\end{array}$ & & & & \\
\hline $3 c$ & 4. Porque realmente me gusta asistir a clase & & & & \\
\hline 1 & $\begin{array}{l}\text { 5. Sinceramente no lo sé, creo que estoy perdiendo el tiempo en el } \\
\text { instituto }\end{array}$ & & & & \\
\hline $3 \mathrm{~b}$ & 6. Por el placer que siento cuando me supero en los estudios & & & & \\
\hline $2 b$ & 7. Para demostrarme que soy capaz de terminar el Ciclo & & & & \\
\hline $2 \mathrm{a}$ & 8. Para conseguir un puesto de trabajo más prestigioso & & & & \\
\hline $3 a$ & $\begin{array}{l}\text { 9. Por el placer que siento cuando descubro cosas nuevas que nunca } \\
\text { había visto antes }\end{array}$ & & & & \\
\hline $2 \mathrm{C}$ & $\begin{array}{l}\text { 10. Porque me permitirá acceder al mercado laboral en el campo que más } \\
\text { me gusta }\end{array}$ & & & & \\
\hline $3 c$ & 11. Porque para mí, el instituto es divertido & & & & \\
\hline 1 & $\begin{array}{l}\text { 12. Antes tenía buenas razones para ir al instituto, pero ahora me pregunto } \\
\text { si vale la pena continuar }\end{array}$ & & & & \\
\hline $3 b$ & $\begin{array}{l}\text { 13. Por el placer que siento cuando consigo uno de mis objetivos } \\
\text { personales }\end{array}$ & & & & \\
\hline $2 \mathrm{~b}$ & 14. Porque cuando hago bien las tareas en clase me siento importante & & & & \\
\hline $2 a$ & 15. Porque quiero "vivir bien" una vez que termine & & & & \\
\hline $3 a$ & $\begin{array}{l}\text { 16. Por el placer que siento al ampliar mis conocimientos sobre los temas } \\
\text { que me interesan }\end{array}$ & & & & \\
\hline $2 \mathrm{c}$ & $\begin{array}{l}\text { 17. Porque me ayudará a tomar una mejor decisión en lo que respecta a } \\
\text { mi orientación profesional }\end{array}$ & & & & \\
\hline $3 c$ & $\begin{array}{l}\text { 18. Por el placer que siento cuando participo en debates con profesores } \\
\text { interesantes }\end{array}$ & & & & \\
\hline 1 & 19. No sé por qué voy al instituto y, sinceramente, no me importa & & & & \\
\hline $3 \mathrm{~b}$ & $\begin{array}{l}\text { 20. Por la satisfacción que siento cuando voy superando actividades } \\
\text { académicas difíciles }\end{array}$ & & & & \\
\hline $2 \mathrm{~b}$ & 21. Para demostrarme que soy una persona inteligente & & & & \\
\hline $2 \mathrm{a}$ & 22. Para poder conseguir, posteriormente, un mejor salario & & & & \\
\hline $3 a$ & $\begin{array}{l}\text { 23. Porque mis estudios me permiten seguir aprendiendo muchas cosas } \\
\text { que me interesan }\end{array}$ & & & & \\
\hline $2 \mathrm{c}$ & $\begin{array}{l}\text { 24. Porque creo que la educación que recibo en el instituto mejorará mi } \\
\text { competencia laboral }\end{array}$ & & & & \\
\hline $3 c$ & 25. Porque me estimula leer sobre los temas que me interesan & & & & \\
\hline 1 & 26. No lo sé, no entiendo qué hago en el instituto & & & & \\
\hline $3 b$ & $\begin{array}{l}\text { 27. Porque las clases me producen satisfacción personal cuando trato de } \\
\text { conseguir lo máximo en mis estudios }\end{array}$ & & & & \\
\hline $2 \mathrm{~b}$ & 28. Porque quiero demostrarme que puedo superar mis estudios & & & & \\
\hline
\end{tabular}

Nota: MD=Muy en desacuerdo; $\mathrm{PA}=$ Poco de acuerdo; $\mathrm{MA}=$ Muy de acuerdo; $\mathrm{TA}=$ Totalmente de acuerdo. 